\title{
Investigation into Machining Accuracy of Micro Circular Pattern Fabricated by Maskless Electrochemical Micromachining
}

\author{
S. Kunar ${ }^{1, *}$, A. P. Tiwary ${ }^{2}$, G. Kibria ${ }^{3}$ and Bh. V. Prasad ${ }^{1}$ \\ ${ }^{I}$ Department of Mechanical Engineering, Aditya Engineering College, Surampalem, India \\ ${ }^{2}$ Department of Mechanical Engineering, Sikkim Manipal Institute of Technology, Sikkim Manipal University, Sikkim, India \\ ${ }^{3}$ Department of Mechanical Engineering, Aliah University, Kolkata, India
}

Received 07 March 2021; Accepted 22 August 2021

\begin{abstract}
Micro circular pattern defines the crucial performance of many micro-industrial components and micro-products. The dimensions, surface quality and location of micro circular pattern directly influence the product life. In maskless electrochemical micromachining (EMM), the maskless substrates are used for the manufacture of many impressions with insulated micro circular patterned tool. One textured tool produces more than twenty-three machined samples. This method is successfully utilized to produce multiple the dimple patterns because of its important benefits like stress and crack free surfaces, material independent of hardness, reusability of the tool and ability to cut the material irrespective of the hardness. The maskless EMM has some distinctive benefits i.e., less machining time and inexpensive. In this paper, the outcome of process variables i.e., electrolyte concentration and frequency on the machining accuracy and depth is investigated. Higher electrolyte concentration and lower pulsed frequency increase the overcut. The combination of lower electrolyte concentration and higher frequency is recommended for high machining accuracy.
\end{abstract}

Keywords: EMM, micro circular pattern, machining accuracy, overcut, depth

\section{Introduction}

Micropatterned surfaces characterize the advancement of micromachining technology for enhancing the performance of manufacturing components. These surfaces can change the thermal, tribological, etc. properties. Many reviewers have evaluated their theories on micro-texturing and microtextured techniques [1-2]. Various types of microscopic effect can be obtained by the different microscopic mechanisms. Surface microtextures retain the lubricating substances in micro-impression and act as lubricant reservoirs. These micro-textures reduce the shear stress in lubricated mating elements. Menezes et al. [3] shows the micro-features of micro-circular pattern used in many engineering fields and applied to the development of tribological properties of automobile components.

Various micro-texturing techniques i.e., abrasive jet machining, laser machining, electro-discharge machining, electrochemical machining, etc. are used for fabrication of different textures. Bao et al. [4], Rajurkar et al. [5] and Zhu et al. [6] presents that electrochemical machining is an advanced machining technique due to several benefits i.e., tool wear, free from residual stresses, cracks and burrs and inexpensive compared to other methods. Electrochemical machining method can dissolve the material by maskless or through-mask method. Natsu et al. [7] proposed the electrolyte jet machining for the generation of dimple pattern with $300 \mu \mathrm{m}$ in diameter. Nouraeiz et al. [8] presents the maskless EMM process in which the anode is kept near to the cathode for generation of my micropattern. Costa et al. [9] uses maskless electrochemical texturing method for generation of dimple arrays with diameter of $220 \mu \mathrm{m}$. Byun et al. [10] proposes the micro- electrochemical machining technique for fabrication of micro-circular patterns with a

*E-mail address: sandip.sandip.kunar@gmail.com

ISSN: 1791-2377 @ 2021 School of Science, IHU. All rights reserved.

doi:10.25103/jestr.144.13 tool electrode diameter of $275 \mu \mathrm{m}$. But it takes more time for individual workpiece fabrication. Kunar et al. [11] fabricates surface structures in different phases to envisage the effect of EMM process variables on the dimple pattern. Thanigaivelan et al. [12] presents the effect of process inputs on overcut using electrochemical micromachining. Mahata et al. [13] uses through-mask electro-chemical micromachining to study the influence of duty ratio on other surface properties and overcut utilizing very thin mask. Kunar et al. [14] shows the impact of EMM process inputs on depth and overcut of dimple pattern have investigated.

The micro circular patterned surface is described by its various arithmetical properties i.e. shape, size, etc. All geometrical properties have lots of significance in tribological applications. Therefore, accurate micro-circular pattern generation is very important factor for the tribological purpose.

A novel method of maskless electrochemical micromachining technique, involving micropattern transfer without maskless workpieces, has been planned for the fabrication of micro-circular pattern with enhanced surface quality using developed electrochemical micromachining system, electrochemical micromachining cell with vertical cross flow system and EMM setup. One masked patterned tool can produce numerous micro-circular patterns. The purpose of the research paper is to explore the influence of process input, especially the influence of electrolyte concentration and pulse frequency on machining accuracy.

\section{Experimental procedure}

The developed setup consists of different sub-components i.e., pulsed power unit, electrolyte flow scheme, machining 
cell, etc., used for experimental research, as shown in Figure 1. EMM cell is a cuboidal shaped box made from corrosion resistance material i.e., Perspex. The cell has diverse précised components i.e., electrolyte circulation arrangement, electrode holders and electrical connections as shown in Figure 2. The restrained vertical cross flow scheme with fixtured devices are developed in the cell. Fixture system is rigid enough to avoid vibration under electrolyte pressure. It is also essential to consider corrosive environment while designing the workpiece and tool fixture mechanisms. So, the use of non-corrosive and electrically non-conductive materials for fabricating workpiece and tool fixtures is generally recommended. Electrolyte is pumped from a tank, lined by the help of corrosion resistance pump, and flows through the job and tool. Spent electrolyte returns to the tank. The hydroxide sludge and electrolysis products cannot accrue in the narrow gap due to the vertical cross flow of electrolyte. All fittings are made from corrosion resistance material i.e., plastics. The SU-8 2150 coating is used to create textured tools on stainless steel plates using a UV exposure approach with a diameter of $170 \mu \mathrm{m}$ and a coating thickness of $225 \mu \mathrm{m}$. The distance between two successive impressions along the periphery is $800 \mu \mathrm{m}$.

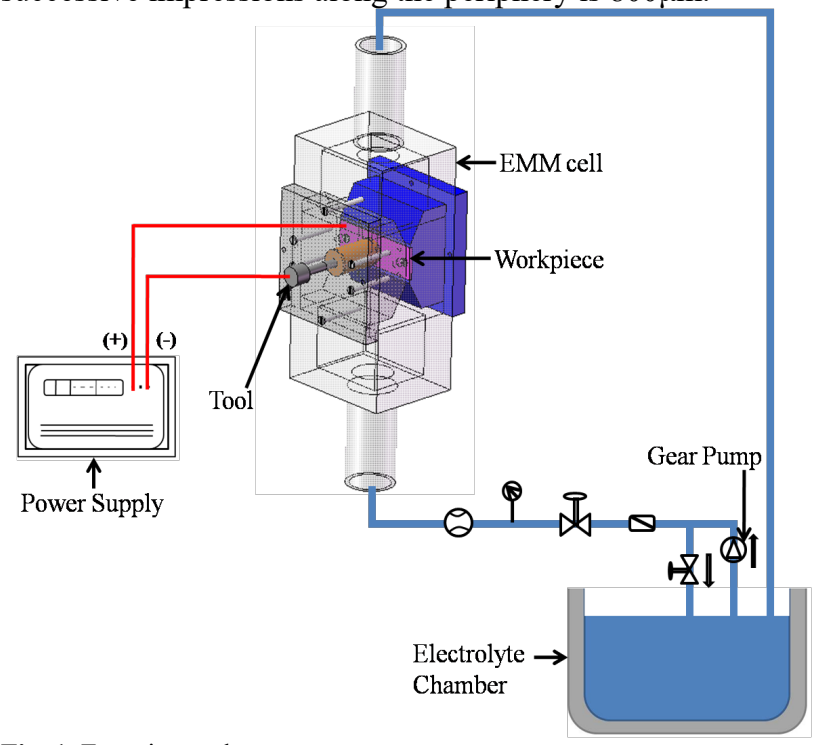

Fig. 1. Experimental setup.

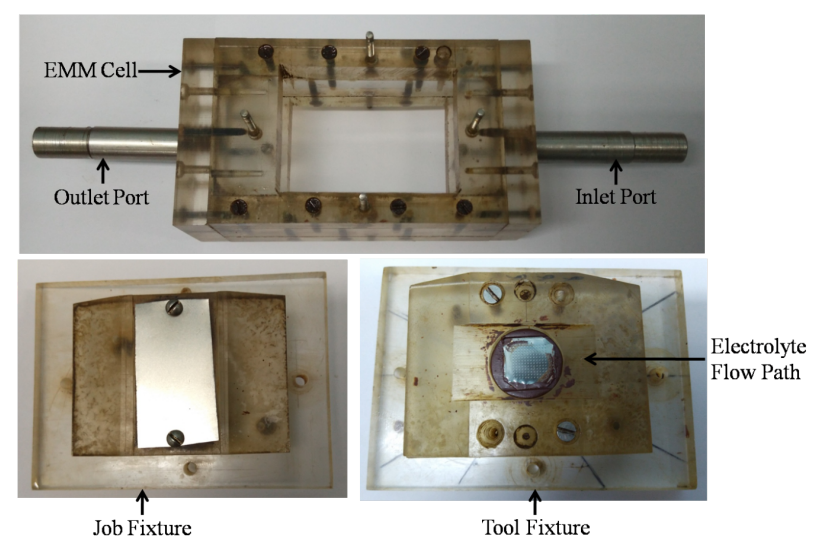

Fig. 2. Machining cell with electrode fixtures.

For effective industrial application, the appropriate maskless EMM process variables are determined for generation of the desired micro circular pattern. Electrolyte concentration and pulsed frequency are significant process inputs for the exploration of machining accuracy of circular pattern. The range of electrolyte concentration and pulsed frequency are typically chosen as 10 to $25 \mathrm{~g} / \mathrm{l}$ and 100 to 190 $\mathrm{kHz}$, respectively. The voltage, duty ratio and current cut off are kept constant, i.e., $12 \mathrm{~V}, 40 \%$ and $3 \mathrm{~A}$, respectively. For experimentation, mixed electrolytes of $\mathrm{NaNO}_{3}(50 \%)$ and $\mathrm{NaCl}(50 \%)$ are favored. The inter electrode gap is $200 \mu \mathrm{m}$. All surface structuring experiments have been conducted for 3 minutes. Electrolyte is distributed during experimentation at a flow rate of $4.5 \mathrm{~m}^{3} / \mathrm{hr}$.

Different process variables are related with machining accuracy and calculated as follows:

Current density

$$
\rho=\frac{\mathrm{I}}{\mathrm{A}}
$$

Where, machining current is I and of machined area is A. Current efficiency

$$
\xi=\frac{\dot{m}}{(A I t / z F)}
$$

Where, $\dot{m}$ is the observed mass material removal rate, $\mathrm{z}$ is valency, $\mathrm{A}$ is atomic weight, $\mathrm{I}$ is machining current, $\mathrm{F}$ is Faraday's constant and time t. Mean micro circular impression diameter,

$$
D=\frac{\sum_{i=1}^{N} D_{i}}{N}
$$

Where, at the $i$ th measurement, the micro-circular impression diameter is $\mathrm{D}_{\mathrm{i}}$.

Mean micro circular impression depth,

$$
H=\frac{\sum_{i=1}^{N} H_{i}}{N}
$$

Where, at the ith measurement, the micro-circular impression depth is $\mathrm{H}_{\mathrm{i}}$.

Standard deviation of micro circular impression diameter,

$$
S_{d}=\sqrt{\sum_{i=1}^{N} \frac{\left(D_{i}-D\right)^{2}}{N-1}}
$$

Where, at the $i$ th measurement, the micro circular impression diameter is $\mathrm{D}_{\mathrm{i}}$.

Standard deviation of micro circular impression depth,

$S_{h}=\sqrt{\sum_{i=1}^{N} \frac{\left(H_{i}-H\right)^{2}}{N-1}}$

Where, at the $i$ th measurement, the micro circular impression depth is $\mathrm{H}_{\mathrm{i}}$.

Diametric taper angle, 


$$
\theta=\tan ^{-1} \frac{D-D_{m}}{2 H}
$$

Here, the total number of impressions is $\mathrm{N}$.

\section{Results and discussion}

To investigate the machining accuracy of circular pattern, different process inputs i.e., electrolyte concentration and pulsed frequency vary for various surface characteristics i.e., current density, current efficiency, mean micro circular impression diameter, mean micro circular impression depth, standard deviation of micro circular impression depth, standard deviation of micro circular impression diameter and diametric taper angle.

\subsection{Effect of electrolyte concentration on micro circular pattern}

To investigate the correlation between electrolyte concentration and machining accuracy, the dimple pattern is fabricated at electrolyte concentration in the range of 10 to $25 \mathrm{~g} / \mathrm{l}$ with the same frequency of $190 \mathrm{kHz}$.

Figure 3 shows the impact of electrolyte concentration on current density and efficiency. The current density and efficiency boost with rising electrolyte concentration because the current flow rises with higher electrolyte concentration and the augmented current raises MRR. The current density and current efficiency increase gradually linear in nature from $10 \mathrm{~g} / 1$ to $25 \mathrm{~g} / 1$ with increased electrolyte concentration.

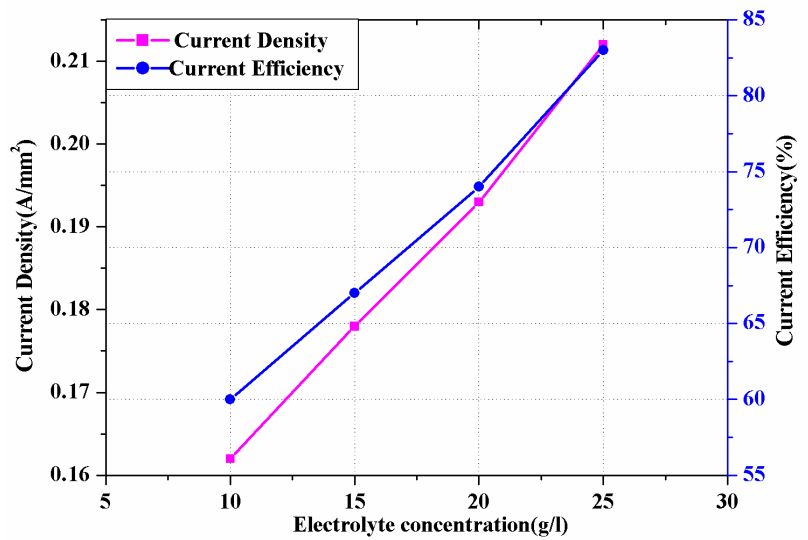

Fig. 3. Variation of current density and current efficiency with electrolyte concentration.

Figure 4 represents the variations of mean micro impression diameter and mean micro impression depth with respect to different electrolyte concentrations of micro circular pattern. The mean micro impression diameter and mean micro impression depth increase continuously with the increase in electrolyte concentrations. It occurs due to the increase of current flux with respect to increased electrolyte concentrations. This increased current flux increases the anodic dissolution efficiency. The mean micro impression diameter amplifies owing to higher stray current affect and the mean micro impression depth increases due to more localization effect.

The impact of electrolyte concentration is shown in Figure 5 on the standard deviations of micro-circular diameter and depth. With rising electrolyte concentration due to higher stray current effect, the standard deviations increase, lowering the precision of machining. Micro- circular depth raises standard deviations due to uncontrolled machining with increased electrolyte concentration.

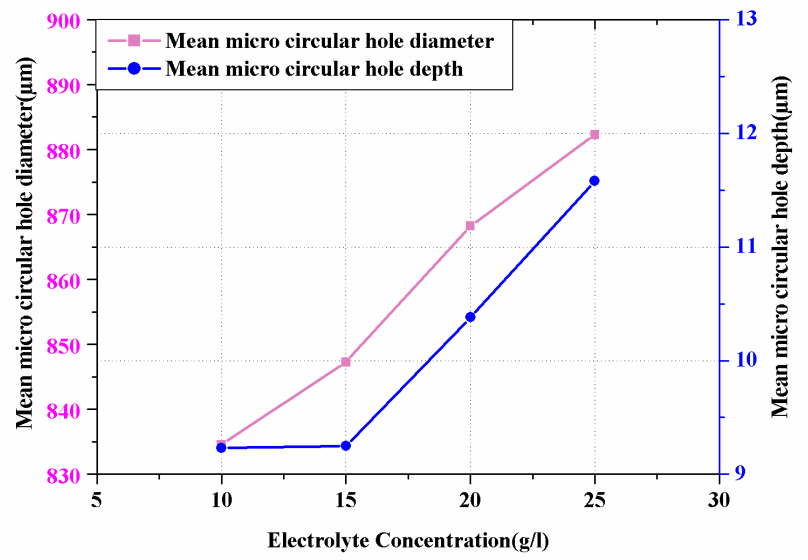

Fig. 4. Variation of $\mathrm{D}$ and $\mathrm{H}$ with electrolyte concentration.

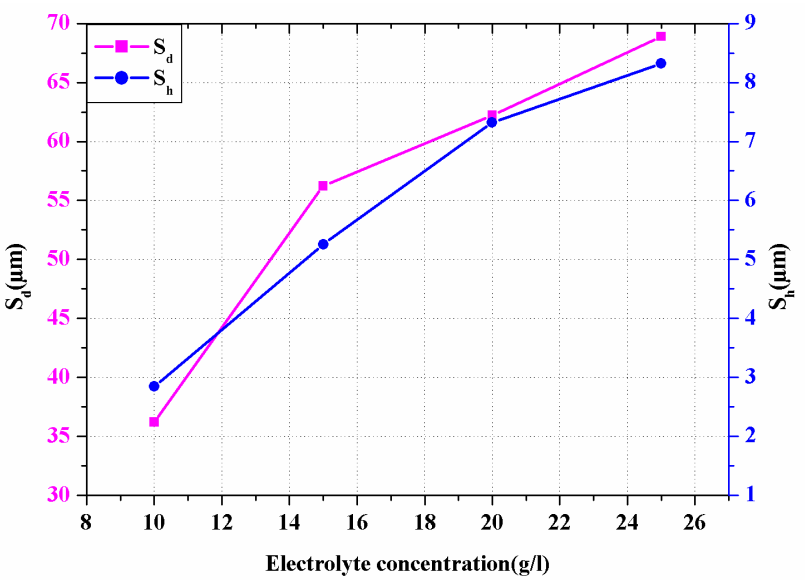

Fig. 5. Variation of $S_{d}$ and $S_{h}$ with electrolyte concentration.

Figure 6 plots the outcome of electrolyte concentration on diametric taper angle. The diametric taper angle augments with increased electrolyte concentration from 10 to $25 \mathrm{~g} / \mathrm{l}$. It occurs due to increase of stray current affect which reduces the machining accuracy from $10 \mathrm{~g} / 1$ to $25 \mathrm{~g} / \mathrm{l}$. It also decreases the localization effect.

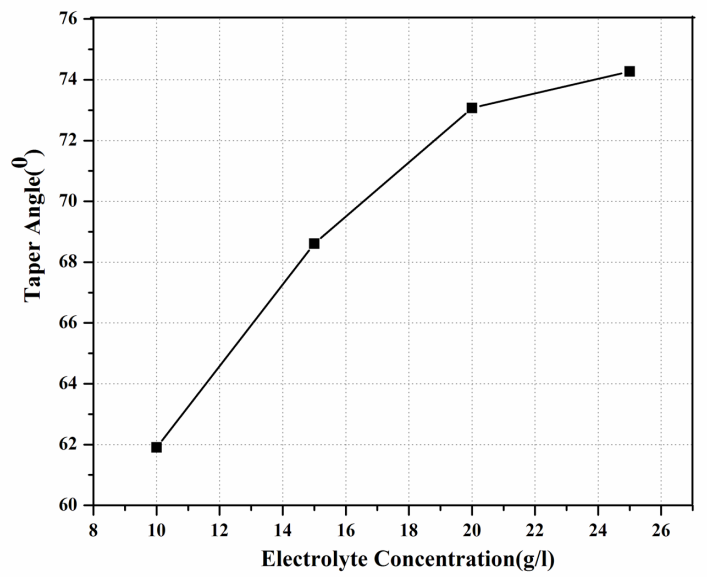

Fig. 6. Variation of diametric taper angle with electrolyte concentration.

3.2 Effect of pulsed frequency on micro circular pattern Pulsed frequency is one more significant input that is correlated with machining accuracy and the patterns are 
generated with varying pulsed frequency from $100 \mathrm{kHz}$ to $190 \mathrm{kHz}$ for the same electrolyte concentration of $10 \mathrm{~g} / \mathrm{l}$.

Figure 7 plots the outcome of frequency on current density and current efficiency for micro circular pattern performed at different pulsed frequency. As the machining time declines at higher frequency, current density and efficiency reduces with rising pulsed frequency. Therefore, the current flow decreases with increased pulsed frequency.

The difference in the mean micro impression diameter and mean micro impression depth of the circular pattern at various pulsed frequency is shown in Figure 8. Due to the availability of more machining time at lower frequency than at higher frequency, it is observed that the mean diameter and mean depth decrease continuously with increased frequency from $100 \mathrm{kHz}$ to $190 \mathrm{kHz}$.

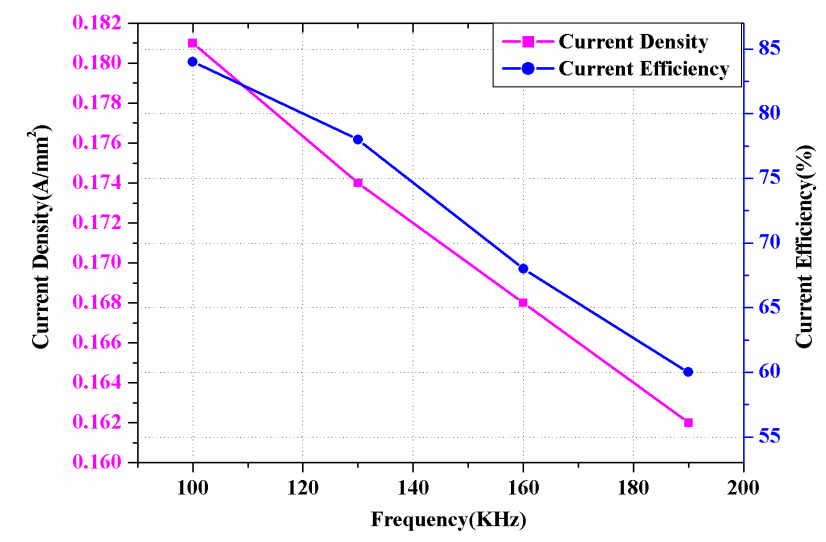

Fig.7. Variation of current density and current efficiency with frequency.

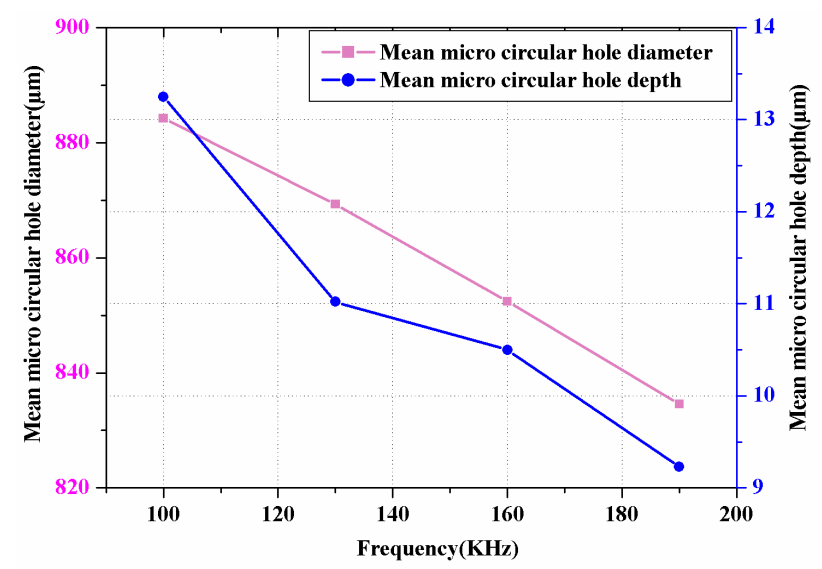

Fig. 8. Variation of on D and H with frequency.

Figure 9 demonstrates the influence of frequency on the standard deviations of micro circular impression diameter and depth at different frequencies. It decreases with increasing pulsed frequency because the overcut decreases for controlled machining from. Therefore, the machining accuracy improves as the frequency rises.

Figure 10 represents the outcome of frequency on diametric taper angle in the range of $100 \mathrm{kHz}$ to $190 \mathrm{kHz}$. With rising frequency, the diametric taper angle declines because of higher machining accuracy for lower stray current effect. The stray current effect is decreased in the higher frequency range as the available machining time is lower, resulting in a reduction of the diameter taper angle.

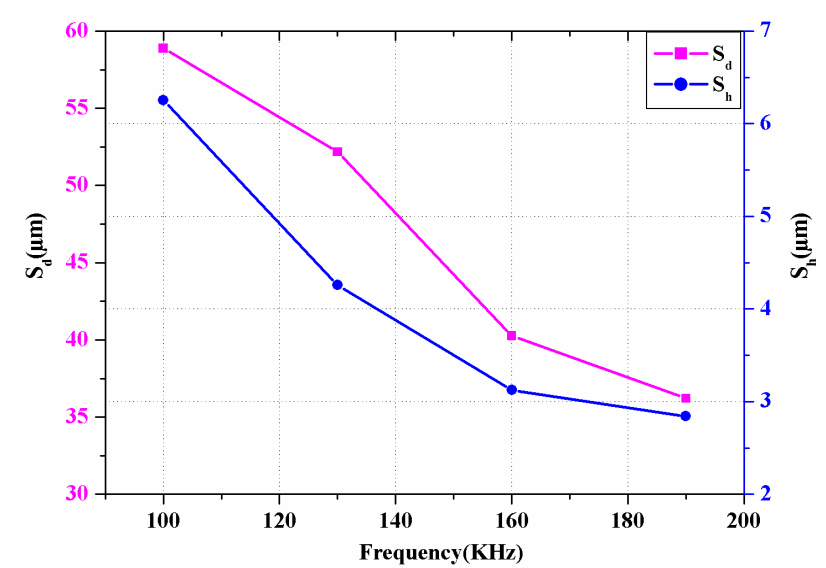

Fig. 9. Variation of $S_{h}$ and $S_{d}$ with frequency.

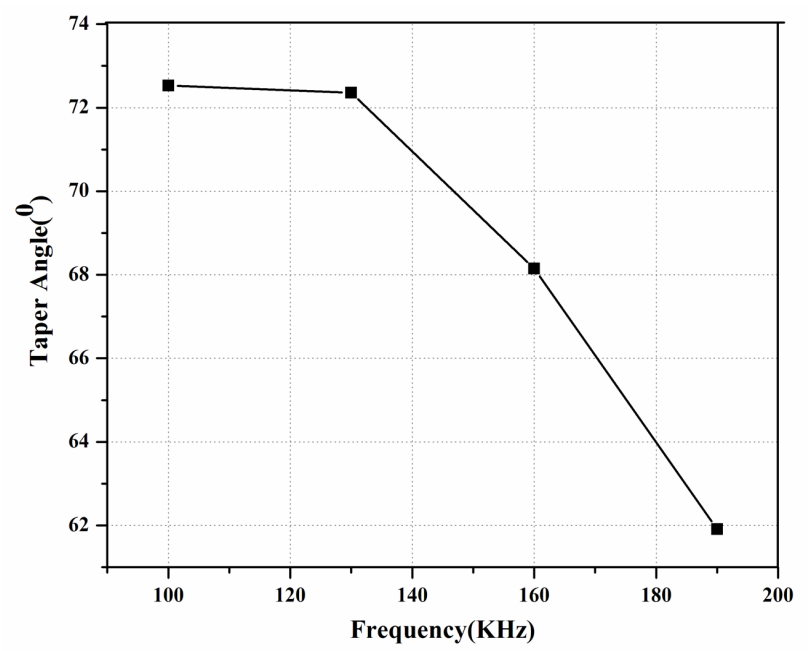

Fig. 10. Variation of diametric taper angle with frequency.

\subsection{Analysis of textured pattern}

In the mask patterning method, the masked tool is used to perform experiments to produce micro circular patterns. To produce more than eighteen machined specimens, this mask has sufficient strength for retaining its capability. Figure 11(a) depicts the patterned tool before texturing. Figure 11(b) illustrates the tool after texturing. After manufacturing multiple machined samples, it will not be damaged. Because it has higher strength to withstand higher speeds, and can adhere to the substrate, it can be reused. Therefore, the SU-8 2150 mask has greater potential to use the technique of maskless electrochemical micromachining to produce many high-quality, low-cost, and short-term micro circular patterns.

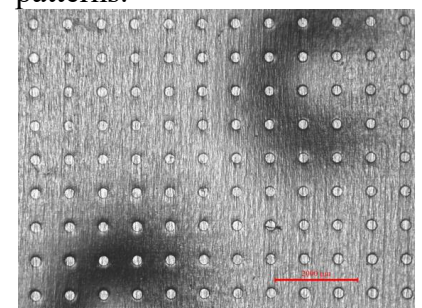

(a)

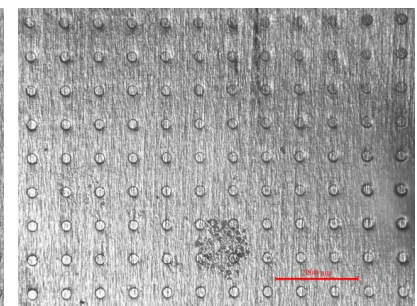

(b)
Fig. 11. Textured tool (a) before texturing (b) after texturing.

Experimental studies have confirmed that both electrolyte concentration and frequency have a significant impact on the size and processing accuracy of the micro 
circular pattern. However, the lower electrolyte concentration and higher frequency are the best combinations to enhance the machining accuracy. Figure 12 displays an image of a micro circular pattern taken by an optical microscope and processed using different parameters, i.e., $10 \mathrm{~g} / \mathrm{l}$ electrolyte concentration and $190 \mathrm{kHz}$ pulse frequency. The blended impact of lower electrolyte concentration and higher frequency allow the fabrication of circular impressions with controllable diameter and depth in circular pattern to get the better machining accuracy. During this process, the current density and current efficiency are lower at lower electrolyte concentration and higher frequency to obtain the controlled machining accuracy in circular pattern. The higher electrolyte concentration and lower frequency etch the wider area with uncontrolled machining accuracy with higher current density and efficiency. Hence, the lower electrolyte concentration and higher frequency are the better parameter combination to enhance machining quality.

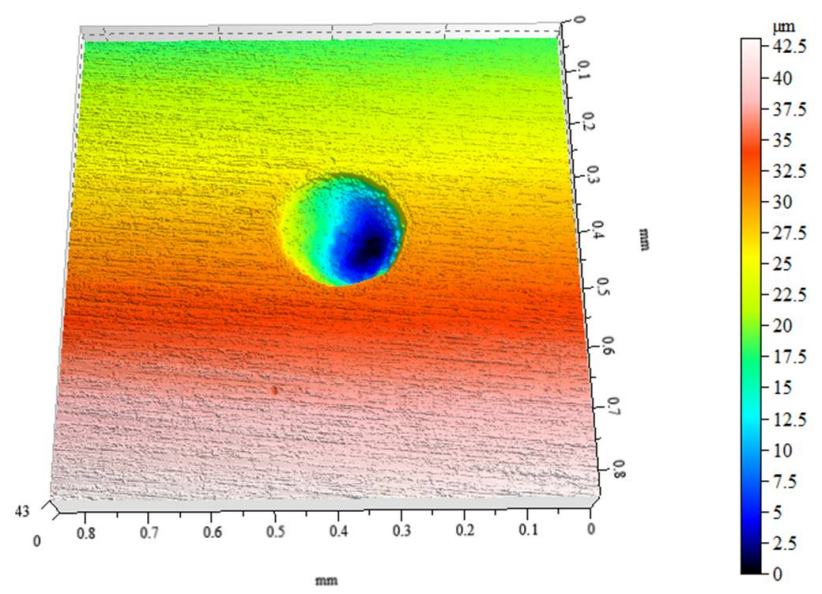

Fig. 13. 3D view and 2D depth profile of micro circular impression.

\section{Conclusions}

The advantages of maskless electrochemical micromachining are maskless substrates with high machining accuracy of circular pattern, a reused textured cathodic tool carrying SU-8 2150 mask and low cost in mass production. Based on the results of investigation, the terminations of this research work can be drawn as follows:

(i) The system presented can achieve higher circular patterned machining accuracy compared to other EMM methods.

(ii) The masked tool can fabricate more than eighteen machined samples with higher machining accuracy.

(iii) For higher machining accuracy, the combination of higher pulsed frequency and lower electrolyte concentration is recommended.

(iv) From the analysis of micrographs, the obtained suitable machining conditions i.e., electrolyte

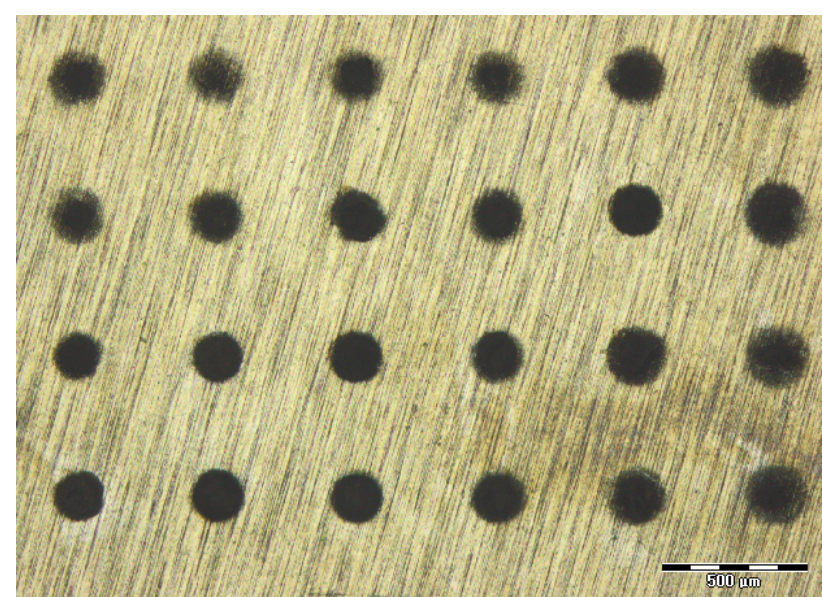

Fig. 12. Regular micro circular pattern

A $3 \mathrm{D}$ view and 2D depth profile of the micro impression are shown in figure 13 . The depth of profile is $33 \mu \mathrm{m}$.

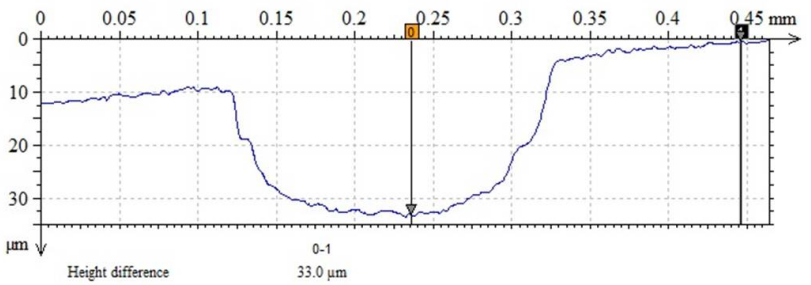

concentration of $10 \mathrm{~g} / \mathrm{l}$ and pulsed frequency of $190 \mathrm{kHz}$ can fabricate the high quality micro circular pattern with higher machining accuracy.

The presented manuscript will be very helpful for better insight of maskless EMM procedure to achieve the improved machining accuracy of circular pattern. Minimization of overcut with lower electrolyte concentration and higher frequency will go a long way towards high machining accuracy of micro products development. The higher machining accuracy of circular pattern is a challenging issue for getting better shape, size and performance characteristics in maskless EMM operation.

This is an Open Access article distributed under the terms of the Creative Commons Attribution License.

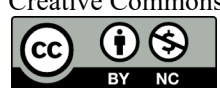

\section{References}

1. A.A.G. Bruzzone, H.L. Costa, P.M. Lonardom, and D.A. Lucca, "Advances in engineered surfaces for functional performance," CIRP Annals-Manufacturing Technology, 2008, Vol. 57, No. 2, pp. 750769.
2. C.J. Evans, and J.B. Bryan, "Structured, textured or engineered surfaces," CIRP Annals - Manufacturing Technology, 1999, Vol. 48, No 2, pp. 541-556.

3. P.L. Menezes, and K.S.V. Kishore, "Influence of die surface textures during metal forming - a study using experiments and simulation," 
Materials and Manufacturing Processes, 2010, Vol. 25, No. 9, pp. $1030-1039$.

4. H.Q. Bao, J.W. Xu, and Y Li, "Aviation-oriented micromachining technology-micro- ECM in pure water," Chinese Journal of Aeronautics, 2008, Vol. 21, No 5, pp. 455-461.

5. K.P. Rajurkar, D. Zhu, J.A. Mcgeough, J. Kozak, and A.D. Silva, "New developments in electro-chemical machining," CIRP Annals Manufacturing Technology, 1999, Vol. 48, No. 2, pp. 567-579.

6. D. Zhu, Z.Y. Xu, and L.S. Zhou, "Trajectory control strategy of cathode in blisk electrochemical machining," Chinese Journal of Aeronautics, 2013, Vol. 26, No 4, pp. 1064-1070.

7. M. Natsu, T. Ikeda, and M. Kunieda, "Generating complicated surface with electrolyte jet machining," Precision Engineering, 2007, Vol. 31, No. 1, pp. 33-39.

8. S. Nouraeiz, and S. Roy, "Electrochemical process for micropattern transfer without photolithography: a modeling analysis," Journal of Electrochemical Society, 2008, Vol. 155, No 2, pp. D97-D103.

9. H.L. Costa, and I.M. Hutchings, "Development of a maskless electrochemical texturing method," Journal of Material Processing Technology, 2009, Vol. 209, No. 8, pp. 3869-3878.
10. J.W. Byun, H.S. Shin, M.H. Kwon, B.H. Kim, and C.N. Chu, "Surface texturing by micro-ECM for friction reduction," International Journal of Precision Engineering and Manufacturing, 2010, Vol. 11, No. 5, pp. 747-53.

11. S. Kunar, and B. Bhattacharyya, "Investigation on surface structuring generated by electrochemical micromachining," Advances in Manufacturing, 2017, Vol. 5, No. 3, pp. 217-230.

12. R. Thanigaivelan, and R.M. Arunachalam, "Experimental study of overcut in electrochemical micromachining for 304 stainless steel," Transactions of the North American Manufacturing Research Institution of the Society of Manufacturing Engineers (NAMRI/SME), 2010, Vol. 38, pp. 253-260.

13. S. Mahata, S. Kunar, and B. Bhattacharyya, "Micro dimple array fabrication by through mask electrochemical micromachining utilizing low-aspect ratio mask," Journal of Electrochemical Society, 2018, Vol. 165, No 3, pp. E129-E137.

14. S. Kunar, and B. Bhattacharyya, "Electrochemical microsurface texturing with reusable masked patterned tool," Engineering Science and Technology, an International Journal, 2018, Vol. 21, No. 5, pp. 1095-1103. 